Wright State University

CORE Scholar

$10-1971$

\title{
Nuclear-Magnetic-Resonance Studies of the Semiconductor-to- Metal Transition in Chlorine-Doped Cadmium Sulfide
}

\author{
Frank D. Adams \\ David C. Look \\ Wright State University - Main Campus, david.look@wright.edu \\ Carlton L. Brown \\ Donald R. Locker
}

Follow this and additional works at: https://corescholar.libraries.wright.edu/physics

Part of the Physics Commons

\section{Repository Citation}

Adams, F. D., Look, D. C., Brown, C. L., \& Locker, D. R. (1971). Nuclear-Magnetic-Resonance Studies of the Semiconductor-to-Metal Transition in Chlorine-Doped Cadmium Sulfide. Physical Review B, 4 (7),

2115-2123.

https://corescholar.libraries.wright.edu/physics/610

This Article is brought to you for free and open access by the Physics at CORE Scholar. It has been accepted for inclusion in Physics Faculty Publications by an authorized administrator of CORE Scholar. For more information, please contact library-corescholar@wright.edu. 
${ }^{2}$ P. Colombino, P. Fiscella, and L. Trossi, Nuovo Cimento 27, 589 (1963).

${ }^{3}$ K. Fujiwara, J. Phys. Soc. Japan 20, 1533 (1965); K. Fujiwara and O. Sueoka, ibid. 21, 1947 (1966); K. Fujiwara, O. Sueoka, and T. Imura, ibid. 24, 467 (1968); D. L. Williams, E. H. Becker, P. Petievich, and G. Jones, Phys. Rev. Letters 20, 448 (1968).

${ }^{4}$ The author wishes to thank P. Osmon, M. Howells, and T. K. Chatterjee of Westfield College, London, for describing their preliminary experiments with the sparkchamber setup, and raising the question how the data should be analyzed.
${ }^{5}$ P. E. Mijnarends, Phys. Rev. 160, 512 (1967); 178, $622(1969)$.

${ }^{6}$ F. M. Mueller and M. G. Priestley, Phys. Rev. $\underline{148}$, 638 (1966).

${ }^{7}$ A. R. Edmonds, Angular Momentum in Quantum Mechanics (Princeton U. P., Princeton, N. J., 1966).

${ }^{8} \mathrm{R}$. Courant and D. Hilbert, Methods of Mathematical Physics (Interscience, New York, 1953), Vol. 1, Chap. 2. ${ }^{9}$ Tables of Integral Transforms, edited by A. Erdelyi (McGraw-Hill, New York, 1954), Vol. 2, p. 314.

${ }^{10}$ Reference 8, p. 163.

\title{
Nuclear-Magnetic-Resonance Studies of the Semiconductor-to-Metal Transition in Chlorine-Doped Cadmium Sulfide $* \dagger$
}

\author{
Frank D. Adams \\ Air Force Flight Dynamics Laboratory, Wright-Patterson AFB, Ohio 45433 \\ David C. Look \\ Physics Department, University of Dayton, Dayton, Ohio 45433 \\ L. Carlton Brown \\ Ohio State University, Columbus, Ohio 43210 \\ Donald R. Locker \\ Aerospace Research Laboratories, Wright-Patterson AFB, Ohio 45433 \\ (Received 12 May 1971)
}

\begin{abstract}
Spin-lattice relaxation times $\left(T_{1}\right)$ and Knight shifts were measured for $\mathrm{Cd}^{113}$ nuclei in 12 CdS crystals doped with various amounts of chlorine. Hall coefficients were measured in order to estimate conduction-electron concentrations. Data were obtained for all samples at $300^{\circ} \mathrm{K}$ and for some highly doped samples at $77,4.2$, and $2.13^{\circ} \mathrm{K}$. Metallic properties were observed in all samples having electron concentrations $n>2 \times 10^{18} \mathrm{~cm}^{-3}$. At $300^{\circ} \mathrm{K}$, we find $1 / T_{1} \propto n$ for nonmetallic samples and $1 / T_{1} \propto n^{2 / 3}$ when samples are metallic. The latter proportionality continues to hold at lower temperatures. The dependence of $T_{1}$ on $n$ becomes increasingly less pronounced at lower temperatures in the nonmetallic samples indicating that the nuclear relaxation becomes at least partially dependent on mechanisms other than conduction electrons, such as spin-diffusion coupling to paramagnetic impurity sites. In the metallic samples, the Knight shift $K \propto n^{1 / 3}$ and the Korringa product is a constant: $T_{1} T K^{2}=3.3 \times 10^{-6} \mathrm{sec}{ }^{\circ} \mathrm{K}$. Both the Knight shift and Korringa product decrease sharply for $n<2 \times 10^{18} \mathrm{~cm}^{-3}$. Our analysis shows that the Mott transition (formation of an impurity conduction band or transition to "free" conduction) occurs in a region $5 \times 10^{17}<n<1.6$ $\times 10^{18} \mathrm{~cm}^{-3}$ and that the impurity conduction band and the CdS conduction band become merged (i.e., the Fermi level crosses into the CdS conduction band) in a region $1.6 \times 10^{18}$ $<n<2.4 \times 10^{18} \mathrm{~cm}^{-3}$.
\end{abstract}

\section{INTRODUCTION}

This paper reports on the experimental nuclearmagnetic-resonance (NMR) behavior of $\mathrm{Cd}^{113}$ nuclei in chlorine-doped CdS. We have measured the spin-lattice relaxation times $\left(T_{1}\right)$ and Knight shifts $(K)$ in CdS:Cl having a wide range in the doping concentration. These data were complemented by measurements on the electrical properties and used to investigate the semiconductor-to-metal transition.

Pure CdS is a 2.5-eV band-gap photoconductor which becomes an $n$-type semiconductor when donor impurities are present. Chlorine is a donor impurity for CdS and thus evidently goes into the crystalline lattice substitutionally for sulfur. ${ }^{1}$ The electrical conductivity increases with impurity concentration and at a rather high level the doping will effect a semiconductor-to-metal transition. This phenomenon may be studied by NMR via the hyperfine interaction with conduction electrons which affects both $T_{1}$ and $\dot{K}$.

Impurity conduction phenomena in the group IV semiconductors and the III-V compounds have been studied by several investigators using NMR. ${ }^{2-5}$ Cadmium oxide is the only II-VI compound reported 
to have been studied by this technique. ${ }^{6}$ The semiconductor -to-metal transition in CdS was investigated by Toyotomi and Morigaki ${ }^{7}$ by directly measuring electrical properties.

\section{REVIEW OF THEORY}

\section{A. Semiconductor-to-Metal Transition}

When CdS is lightly doped with chlorine, a shallow degenerate impurity level is formed about $0.03 \mathrm{eV}$ below the bottom of the conduction band. ${ }^{8}$ Except at very low temperatures, donor electrons may be thermally excited to the conduction band and CdS exhibits semiconductor properties. As the number of impurity atoms is increased, a concentration is attained that allows some spatial overlap of the donor ground-state wave functions; this lifts the degeneracy of the impurity state. At a slightly higher impurity concentration, delocalization of the electrons occurs without excitation to the CdS conduction band.

The formation of an impurity conduction band has been studied by Mott $^{9}$ who showed that this condition should occur at a critical concentration $N_{c} \simeq\left(0.25 / a_{i}\right)^{3}$ where $a_{i}$ is the impurity Bohr radius; for CdS, $N_{c} \simeq 9 \times 10^{17} \mathrm{~cm}^{-3}$. A further increase in impurity concentration will broaden the impurity conduction band until it finally merges with the CdS conduction band (i.e., the Fermi level crosses into the conduction band) at a concentration $N_{\mathrm{cb}} \approx(1 / 4 \pi) a_{i}^{-3} \simeq 4.5 \times 10^{18} \mathrm{~cm}^{-3}$; this completes the semiconductor-to-metal transition. ${ }^{10}$ We will refer to the region $N<N_{c}$ as "semiconducting," $N_{c}<N<N_{\mathrm{cb}}$ as "quasimetallic," and $N>N_{\mathrm{cb}}$ as "metallic."

The electrical properties, including the Hall coefficient, of CdS: $\mathrm{Cl}$ are reported by Toyotomi and Morigaki to be highly dependent on the temperature in the semiconductive region, weakly temperature dependent in the quasimetallic region, and almost independent of temperature in the metallic region. ${ }^{7}$

\section{B. Spin-Lattice Relaxation}

The $\mathrm{Cd}^{113}$ spin-lattice relaxation in CdS is usually due to an interaction with either conduction electrons or with paramagnetic impurity sites. ${ }^{11}$ If the $T_{1}$ is due to $s$-state conduction electrons, the coupling is mainly via the hyperfine scalar contact interaction. The Hamiltonian may be written ${ }^{12}$ as

$$
\mathcal{H}_{1}=-\frac{8}{3} \pi \gamma_{e} \gamma_{n} \hbar^{2} \sum_{j l} \overrightarrow{\mathrm{I}}_{j} \cdot \overrightarrow{\mathrm{S}}_{l} \delta\left(\overrightarrow{\mathrm{r}}_{j l}\right)
$$

where $\gamma_{2}$ and $\gamma_{n}$ are the electron and nuclear gyromagnetic ratios, respectively, $\hbar$ is Planck's constant divided by $2 \pi$, and $\overrightarrow{\mathrm{I}}_{j}, \overrightarrow{\mathrm{S}}_{l}$, and $\overrightarrow{\mathrm{r}}_{j l}$ are, respectively, the $j$ th nuclear spin, the $l$ th electron spin, and the vector distance between them.
Using the nuclear Zeeman energy as a zero-order Hamiltonian, $T_{1}$ may be calculated using standard time-dependent perturbation theory. In semiconductors, the conduction electrons are generally nondegenerate and the calculation yields ${ }^{12}$

$$
1 / T_{1}=\frac{32}{9} \gamma_{e}^{2} \gamma_{n}^{2} n V^{2}\left\langle\left|\phi_{e}(0)\right|^{2}\right\rangle^{2}\left[2 \pi\left(m^{*}\right)^{3} k T\right]^{1 / 2},
$$

where $n$ is the conduction-electron density, $V$ the sample volume, $m^{*}$ the effective mass of a conduction electron, $k$ Boltzmann's constant, $T$ the temperature, and $\left\langle\left|\phi_{e}(0)\right|^{2}\right\rangle$ the electronic probability density at the nucleus averaged over all energy states (normalized in sample volume $V$ ). It should be noted that $T_{1}$ is independent of the NMR frequency (field) and that $T_{1} \propto n^{-1} T^{-1 / 2}$.

In metals, the electrons are usually degenerate (except at very high temperature). For this case only electrons with energies on the Fermi surface can cause spin transitions. A detailed perturbation calculation, using Eq. (1), yields ${ }^{12}$

$$
1 / T_{1}=\frac{64}{9} \pi^{3} \gamma_{e}^{2} \gamma_{n}^{2} \hbar^{3}\left\langle\left|\phi_{F}(0)\right|^{2}\right\rangle^{2} \rho^{2}\left(E_{F}\right) k T,
$$

where $\rho\left(E_{F}\right)$ is the electron density of states at the Fermi level $E_{F}$, and $\left\langle\left|\phi_{F}(0)\right|^{2}\right\rangle$ the electronic density at the nucleus averaged over states on the Fermi surface (normalized in sample volume). For free electrons, we have

$$
\rho\left(E_{F}\right)=\frac{\left(3 n / 8 \pi^{4}\right)^{1 / 3}}{\hbar^{2}} m^{*} V,
$$

where $V$ is the sample volume. Here again $T_{1}$ is independent of the NMR frequency, but for degenerate electrons $T_{1} \propto n^{-2 / 3} T^{-1}$.

A second applicable spin-lattice relaxation mechanism involves an interaction with paramagnetic impurity sites. Although CdS is a diamagnetic solid, it is possible to have paramagnetic sites scattered throughout the lattice owing to impurities or dislocations. The interaction is a dipole-dipole coupling between the nuclear spin and the unpaired electron of a paramagnetic ion.

In a material such as $\mathrm{CdS}$, the electronic levels are broadened more than the nuclear splitting. Although there are no "exchange flips" between the electron and the nuclear spin, the electron is flipping owing to "electron spin-lattice relaxation." Changes in $S_{z}$ cause magnetic fluctuations at the nuclear site and those Fourier components at the Larmor frequency induce nuclear transitions. The $T_{1}$ is given by ${ }^{13}$

$$
\frac{1}{T_{1}}=\frac{2}{5}\left(\gamma_{n} \gamma_{e} \hbar\right)^{2} \frac{S(S+1)}{r^{6}} \frac{\tau}{1+(\omega \tau)^{2}},
$$

where $\omega$ is the NMR frequency and $\tau$ is usually the longitudinal electron relaxation time. The angular dependence has been averaged in Eq. (4).

The $r^{-6}$ factor in Eq. (4) makes the direct relaxation process extremely sensitive to range. It 
would appear that those nuclei very close to the paramagnetic site are most easily relaxed. Although this is true, these nuclei are usually also subjected to a large static magnetic field from the paramagnetic ion and this shifts their resonance frequency by a considerable amount. As a result these spins are essentially isolated from the rest of the spin system. Only those nuclei beyond a critical range $r=b$ can communicate with each other via the spin-spin interaction. For this reason, $b$ is called the "spin-diffusion barrier radius."

The process of spin diffusion can be described by a diffusion equation of the form

$$
\frac{\partial \theta}{\partial t}=D \Delta \theta
$$

where $\theta$ is the spin temperature at a point. $D$ is a diffusion constant given roughly by ${ }^{14}$

$$
D \simeq a^{2} / 50 T_{2},
$$

where $a$ is the lattice spacing and $T_{2}$ is the spinspin relaxation time.

When the diffusion barrier radius $b$ is small, a region just beyond $b$ will be strongly relaxed by the paramagnetic center. The remainder of the spin system will tend to equilibrium via the spin-diffusion process. Blumberg ${ }^{13}$ has calculated $T_{1}$ for this case and found

$$
1 / T_{1}=8.5 N_{p}\left(C D^{3}\right)^{1 / 4},
$$

where $C=\frac{1}{5}\left(\gamma_{n} \gamma_{e} \hbar\right)^{2} S(S+1) \tau /\left[1+(\omega \tau)^{2}\right]$ and $N_{p}$ is the density of paramagnetic impurity sites. He denoted this as diffusion-limited relaxation.

When $b$ is large, the direct relaxation process is weaker and the entire spin system (outside radius b) can remain in self-thermal equilibrium. Blumberg called this the "rapid-diffusion" case and found

$$
1 / T_{1}=\frac{4}{3} \pi N_{p} C b^{-3} .
$$

This equation is valid for $b \gg(C / D)^{1 / 4}$. If $b$ $\ll(C / D)^{1 / 4}$, then Eq. (7) may be used. ${ }^{15}$

For both diffusion-limited and rapid-diffusion relaxation, the $T_{1}$ is frequency dependent if $\omega \tau$ is of the order of 1 or larger; there may be additional frequency dependence in $\tau$ or $b$. The temperature dependence of $T_{1}$ enters via the electron spin-lattice relaxation time $\tau$ and also possibly through $b$. In general, $1 / \tau$ is an increasing function of the temperature. A plot of $T_{1}$ versus $T$ is usually characterized by a minimum in $T_{1}$ at a temperature where $\omega \tau \approx 1$. At lower temperatures $T_{1}$ is frequency dependent, whereas at higher temperatures the frequency dependence is quenched.

\section{Knight Shift}

The NMR Knight shift ${ }^{16}$ is generally associated with metals and is caused by magnetic polarization of the conduction electrons. Zhogolev has calculated the Knight shift for nondegenerate electrons in a semiconductor; however, he estimates that it is several orders of magnitude smaller than in a metal. ${ }^{17}$

For degenerate conduction electrons, the Knight shift $K$ is obtained by a first-order perturbation calculation using Eq. (1) ${ }^{18}$ :

$$
K \equiv \Delta H / H_{0}=\frac{8}{3} \pi\left\langle\left|\phi_{F}(0)\right|^{2}\right\rangle V \chi_{e},
$$

where $\chi_{e}$ is the electronic susceptibility, which for a free-electron gas is

$$
\chi_{e}=3 \hbar^{2} \gamma_{e}^{2} n / 8 E_{F} \text {, }
$$

where $E_{F}$ is the Fermi energy. By combining Eqs. (9) and (10) and using $E_{F}=\left(\hbar^{2} / 2 m\right)\left(3 \pi^{2} n\right)^{2 / 3}$, the Knight shift is

$$
K=(8 / 9 \pi)^{1 / 3} \gamma_{e}^{2} m^{*} V\left\langle\left|\phi_{F}(0)\right|^{2}\right\rangle n^{1 / 3} .
$$

The Knight shift is independent of temperature and magnetic field and is proportional to the cube root of the electron concentration.

Combining Eqs. (3) and (11) we obtain the socalled Korringa product ${ }^{19}$

$$
T_{1} T K^{2}=\hbar \gamma_{e}^{2} / 4 \pi k \gamma_{n}^{2} \text {. }
$$

This relationship is valid when the conduction electrons are degenerate and, for a given nucleus, depends only upon their $g$ value.

At low temperatures, both the Knight shift and Korringa product should decrease markedly below the $N_{\mathrm{cb}}$ transition concentration since the electrons are trapped in a spatially limited impurity conduction band. (The impurity conduction band does not occupy the entire volume of the sample; this is discussed in more detail later.) Furthermore, at high temperatures, the electrons would be nondegenerate in the conduction band, again decreasing $K$ and $T_{1} T K^{2}$.

\section{EXPERIMENTAL PROCEDURE}

A. Sample Preparation

Twelve samples of CdS:Cl were selected for experimentation. These are designated samples $A-L$. Sample $A$ was an undoped control sample with a room-temperature $T_{1}$ in excess of $200 \mathrm{sec}$. Each successive letter after $A$ denotes a sample with a shorter $T_{1}$ (higher chlorine-impurity content) up to sample $L$ with a room-temperature $T_{1} \approx 0.3 \mathrm{sec}$.

Samples $A-F$ were grown by the vapor-deposition method. Chlorine doping was accomplished by adding small amounts of $\mathrm{CdCl}_{2}$ to the furnace charge. Samples $J$ and $K$ were also vapor grown; however, with these samples the doping was effected by using a chlorine atmosphere in the furnace. Samples $G$, $H, I$, and $L$ were melt grown under pressure with 
$\mathrm{CdCl}_{2}$ added to the furnace charge to provide doping.

\section{B. NMR Measurements}

A Varian V-4200B wide-line NMR spectrometer was used to perform $T_{1}$ and Knight-shift measurements. Data were obtained on all samples at $300{ }^{\circ} \mathrm{K}$ and for some highly doped samples at 77, 4. 2, and $2.13^{\circ} \mathrm{K}$. A two-chamber Pyrex Dewar, to which the cross-coil Varian probe could be clamped, was used with liquid nitrogen and helium at the lower temperatures. The $2.13^{\circ} \mathrm{K}$ temperature was achieved by vacuum pumping on liquid helium.

The spin-lattice relaxation-time measurements were made using the direct saturation-recovery method. The $\mathrm{Cd}^{113}$ magnetization was saturated at the NMR field $H_{0}$ using a large rf field. The main magnetic field was then adjusted 10-25 G off resonance to allow partial recovery of the magnetization. At a preset time, the magnetization was "sampled" by allowing the magnetic field to sweep through the resonance while observing the NMR dispersion signal. The signal height is proportional to the magnetization that has recovered during the off-resonance time period. The $z$ component of the magnetization $M_{z}(t)$ is

$$
M_{z}(t)=M_{\infty}\left(1-e^{-t / T_{1}}\right),
$$

where $M_{\infty}$ is the equilibrium value of the magnetization. Measurements were made for successively increasing values of the time $t$. The value of $T_{1}$ was found by measuring the slope $1 / T_{1}$ of a semi$\log$ plot of $M_{\infty}-M_{\boldsymbol{z}}(t)$ versus $t$. The average error of a measured $T_{1}$ was about $10 \%$.

Knight-shift measurements were obtained by determining the difference in resonance field between a given sample and the undoped CdS sample $A$. No Knight shifts were observed in the lightly doped samples $B-F$; however, resonance shifts were observed in samples $G-L$. The error in measurement was estimated to be about $0.05 \mathrm{G}$ at a resonance field of $8460 \mathrm{G}$.

All of the Knight-shift measurements and a majority of the $T_{1}$ data were reported at an $8-\mathrm{MHz}$ resonant frequency $\left(H_{0} \simeq 8460 \mathrm{G}\right)$. A few $T_{1}$ mea surements were made at $4 \mathrm{MHz}\left(H_{0} \simeq 4230 \mathrm{G}\right)$ in or der to determine the frequency dependence (if any) of this parameter.

\section{Hall Measurements}

Measurements of the Hall coefficient were made on each sample as a function of temperature using a 10-kG magnetic field. These data were used to estimate the conduction-electron concentration. ${ }^{20}$ Samples were cut into small rectangular paralle1epipeds with typical dimensions of $1.0 \times 0.5 \times 0.4$ $\mathrm{cm}$. Electrical leads were attached to each sample using an ultrasonic soldering iron and indium

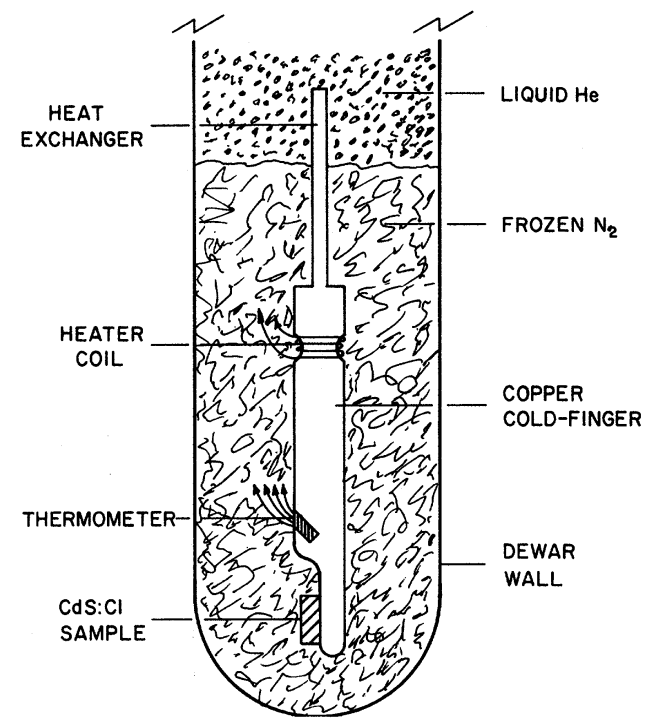

FIG. 1. Simplified drawing of cold-finger device.

solder. A five-lead configuration ${ }^{20}$ was employed to minimize the problem of contact resistance.

Hall measurements were made in the $100^{\circ} \mathrm{K}$ temperature regime using a Varian model V4540 variable-temperature controller (nitrogen gas flow). Liquid $\mathrm{N}_{2}$ and $\mathrm{He}$ were used for the 77 and $4.2^{\circ} \mathrm{K}$ measurements, respectively.

On one particular sample of ' $\mathrm{CdS}: \mathrm{Cl}$, it was necessary to obtain Hall measurements at, temperatures between 4.2 and $77^{\circ} \mathrm{K}$. This required the design and construction of a cold-finger variabletemperature device.

A simplified diagram of the cold-finger device is shown in Fig. 1. The body is a $\frac{3}{8}$-in. -diam, 4-in. long copper rod with a side-flat surface on one end. The CdS:Cl sample was attached to the flat surface with thermally conducting cement. The cold finger was fitted with a thermometer and heating coils. The heat exchanger is a 3 -in. length of No. 12 copper wire. In operation, the cold finger is submerged in liquid nitrogen with about one-half of the heat exchanger above the surface. Liquid helium is then added. This cools and freezes the nitrogen. Solid nitrogen is a relatively poor heat conductor so that the main heat leak is through the copper to the heat exchanger. A heater coil is used to maintain a preselected temperature on the lower part of the cold finger. The temperature is measured with a germanium resistance thermometer. Tests were conducted, by replacing the CdS:Cl sample with a second thermometer, to show that the cold finger does maintain a uniform temperature over the length below the heater coil.

While this system works well, the high boil-off rate of liquid helium makes it most practical when a recovery system is available. This rate can be 


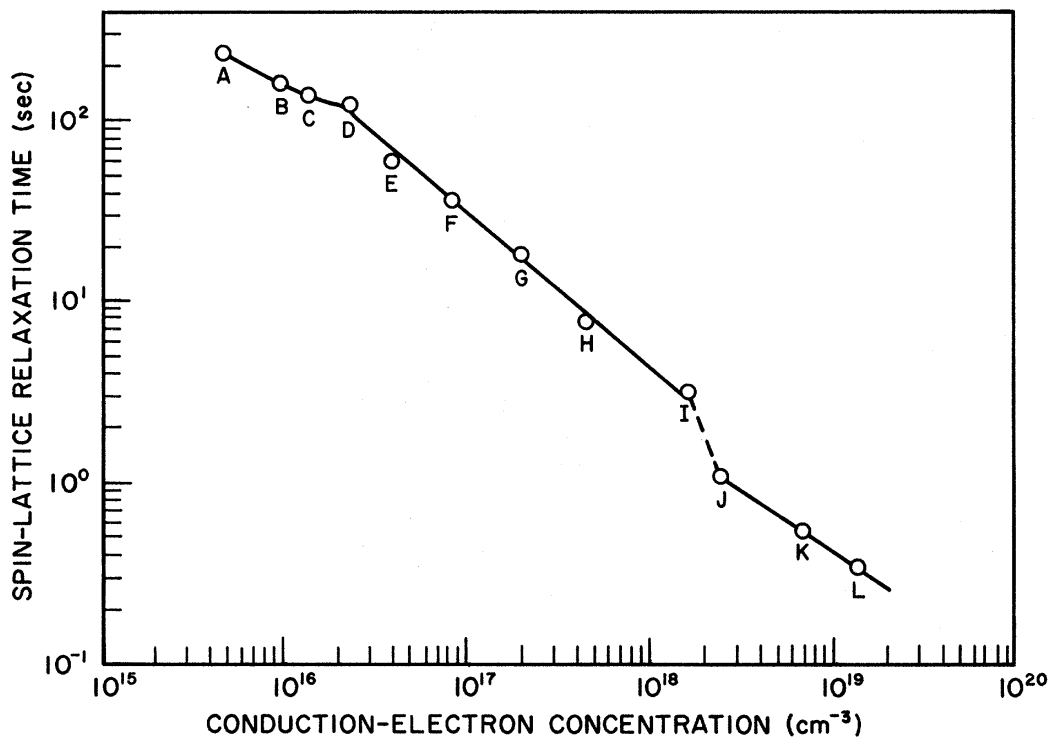

FIG. 2. Spin-lattice relaxation time versus conduction-electron concentration at $300^{\circ} \mathrm{K}$.

decreased by raising the nitrogen level on the heat exchanger or by decreasing the diameter of the heat exchanger. It should be cautioned, however, that this causes the system to be somewhat sluggish in terms of coming to an equilibrium temperature. This is particularly true below $20^{\circ} \mathrm{K}$. Since only a few measurements were needed for this research, no attempt was made to optimize the cold-finger design. It should be noted that, with minimal modifications, this device may be used for NMR measurements.

Hall measurements were secured on all samples between 100 and $350^{\circ} \mathrm{K}$. Data were taken at $4.2^{\circ} \mathrm{K}$ on the heavily doped samples $I, J, K$, and $L$.

Similar measurements were attempted at $4.2^{\circ} \mathrm{K}$ on samples $G$ and $H$ but could not be attained because of large magnetoresistive voltages. The resistivity of samples $G$ and $H$ was measured at $4.2{ }^{\circ} \mathrm{K}$ and estimates of the Hall coefficient were made. These were based on an estimate of the maximum Hall voltage which would not be detected by our apparatus and also by typical values of the Hall mobility published in the literature. ${ }^{7,21}$

Hall measurements were made on sample $I$ be tween 4.2 and $77^{\circ} \mathrm{K}$. The Hall coefficient for this sample did not decrease monotonically with temperature as it did in samples $A-H$ and was not independent of temperature as it was for samples $J, K$, and $L$.

\section{RESULTS AND DISCUSSION}

Figure 2 shows the variation of $T_{1}$ with conduction-electron concentration at $300^{\circ} \mathrm{K}$. These measurements were secured using an $8-\mathrm{MHz}$ resonance. Each datum point was obtained for a different sample $A-L$. In a region $2 \times 10^{16}<n<1.6 \times 10^{18} \mathrm{~cm}^{-3}$, $T_{1}$ is nearly proportional to $n^{-1}$. Equation $2 \mathrm{sug}-$ gests that the conduction electrons are nondegenerate and that samples $D-I$ are nonmetallic. For $n>2.4 \times 10^{18} \mathrm{~cm}^{-3}$, the slope changes to $-\frac{2}{3}$.

From Eq. (3) the conduction electrons are degenerate and samples $J, K$, and $L$ appear to have metallic character. These data indicate that a transition occurs near $n \approx 2 \times 10^{18} \mathrm{~cm}^{-3}$.

For the very lightly doped samples $A, B$, and $C$, the data fall below the $n^{-1}$ line. This suggests that another mechanism such as an interaction with paramagnetic impurity. sites is competing with the conduction electrons to relax the nuclear-spin system.

Measurements on $T_{1}$ at $300^{\circ} \mathrm{K}$ using a $4-\mathrm{MHz}$ resonance support the above conclusions. No frequency dependence was detected in samples $D-L$ which is consistent with spin-lattice relaxation by conduction electrons. However, the $4-\mathrm{MHz} T_{1}$ in samples $A-C$ is slightly lower than at $8 \mathrm{MHz}$ although the change is only about $20 \%$.

In Fig. 3, 8- MHz $T_{1}$ data are shown for 77 and $4.2{ }^{\circ} \mathrm{K}$. Note that samples $J-L$ are still exhibiting metallic properties with $T_{1} \propto n^{-2 / 3}$. With the inclusion of these measurements it is possible to be more specific about the semiconductor-to-metal transition. Note that the electron concentration in sample $I$ is independent of the temperature, yet it does not fall on the $n^{-2 / 3}$ line for metals. This suggests that sample $I$ is quasimetallic and that the transition to sample $J$ corresponds to the merging of the impurity conduction band with the CdS conduction band ( $N_{\mathrm{cb}}$ transition). On the other hand, the electron concentration in sample $H$ is highly dependent on temperature. Thus, samples $H$ and $I$ appear to bracket the Mott transition or the formation of the impurity conduction band ( $N_{c}$ transition).

Below the $N_{\mathrm{cb}}$ transition, the low-temperature 


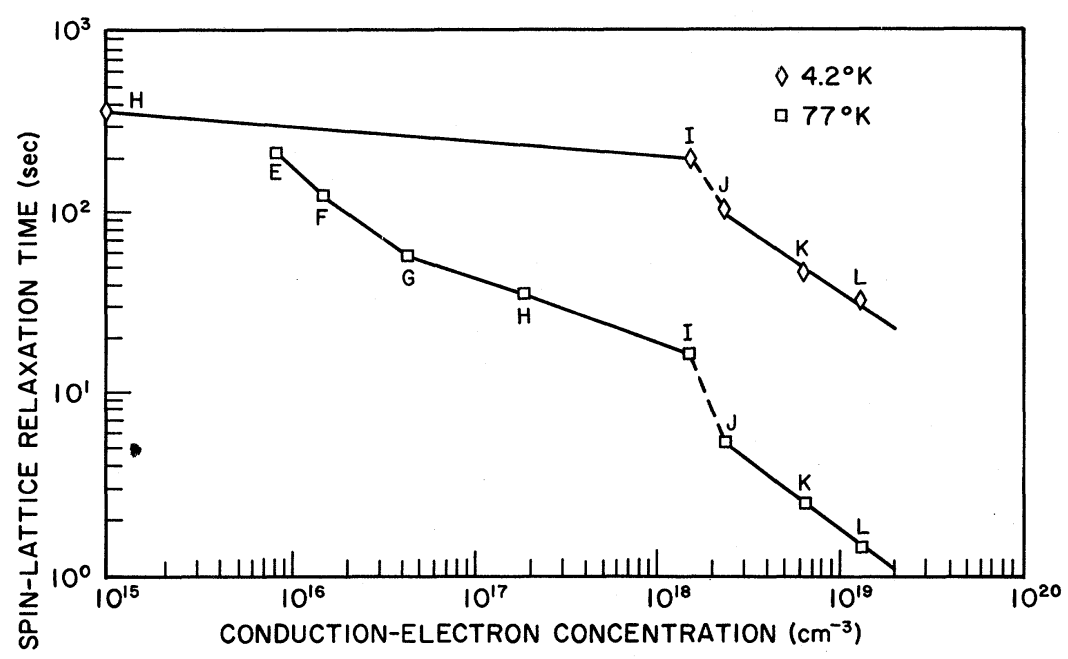

FIG. 3. Spin-lattice relaxation time versus conduction-electron concentration at 77 and $4.2^{\circ} \mathrm{K}$.

spin-lattice relaxation mechanism is a much weaker function of the conduction-electron concentration than was found at $300^{\circ} \mathrm{K}$. This is reasonable since fewer electrons gain the conduction band at low temperatures. Thus, the $T_{1}$ is probably due to paramagnetic impurity centers. This subject will be further discussed later in the paper.

Equation (3) suggests an interesting method of displaying the $T_{1}$ data for the more heavily doped samples. Figure 4 is a plot of $\log T_{1}$ versus $\log \left(n^{2 / 3} T\right)$ for samples $I-L$. Except for those points associated with sample $I$, the data fall near a straight line with a slope of -1 . This further substantiates that the conduction electrons in samples $J-L$ are degenerate and that these samples are basically metallic in character. The data points for sample $I$ fall above the line in Fig. 4. This denotes a change (weakening) in the relaxation mechanism and supports the idea that samples $I$ and $J$ bound the $N_{\mathrm{cb}}$ transition.

Measurements on $T_{1}$ at the lower temperatures made with a 4-MHz resonance are again consistent with the above conclusion. No frequency dependence was noted for samples $J, K$, and $L$ at $77^{\circ} \mathrm{K}$. A slight frequency dependence was detected at $4.2^{\circ} \mathrm{K}$; however, this was a decrease in the measured $T_{1}$ by less than $20 \%$. Paramagnetic impurities may begin to compete with the conduction electrons to relax the spin system at very low temperatures, but the data as plotted in Fig. 4 strongly suggest that interaction with conduction electrons is the predominant spin-lattice relaxation mechanism.

Below the $N_{c b}$ transition, the frequency dependence of $T_{1}$ increases with decreasing temperature. A $20 \%$ decrease in $T_{1}$ is typical for a $4-\mathrm{MHz}$ resonance at $77^{\circ} \mathrm{K}$. At $4.2^{\circ} \mathrm{K}$, the $T_{1}$ decreases by a factor of 2 for samples $H$ and $I$. This might be expected for sample $H$ since at $4.2^{\circ} \mathrm{K}$ very few electrons can gain the conduction band and the $T_{1}$ should be primarily due to paramagnetic impurities. On the other hand, the donor electrons in sample $I$ are delocalized and are free carriers in an impurity conduction band. Apparently the spin-lattice interaction with electrons in an impurity conduction band differs from the interaction with electrons in the CdS conduction band. This is not surprising when one considers that impurity conduction electrons do not have access to the entire volume of a sample. The impurity conduction band may be pictured as an interconnecting maze of tunnels between donor impurity sites.

Two models will be discussed; either one provides for a frequency-dependent $T_{1}$ in sample $I$. Additional data are required to validate or reject either of these models.

The first model is due to Jerome, Ryter, and

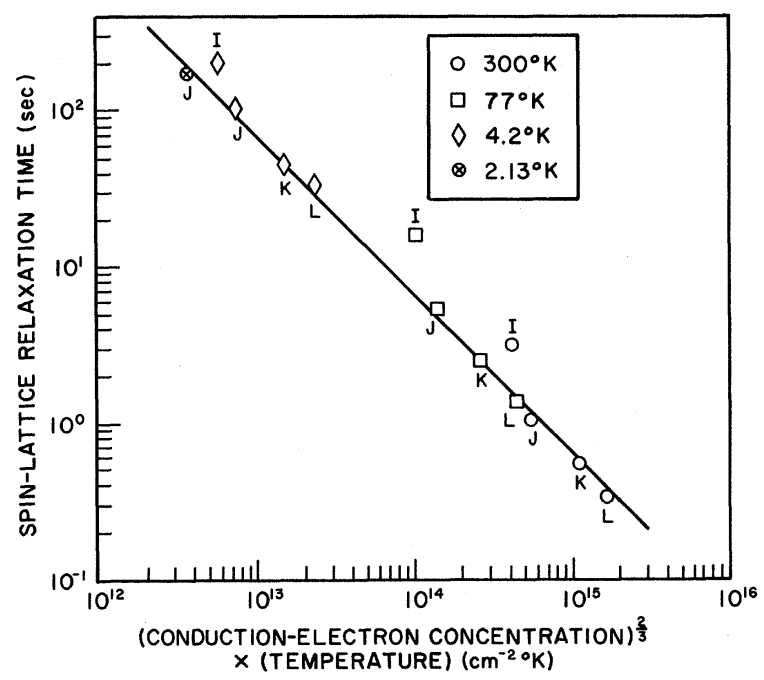

FIG. 4. Spin-lattice relaxation time versus (conduction-electron concentration) $)^{2 / 3} \times$ temperature. 
Winter. ${ }^{22}$ They assume that the nuclear spins in or near impurity-band tunnels are strongly relaxed by conduction electrons. It is further assumed that the remaining spins are relaxed by rapid spin diffusion. However, spin diffusion away from the tunnel regions is inhibited since spins which are inside the tunnels will experience a Knight shift due to polarization of the conduction electrons; those spins which are well outside will be unshifted. To be more precise, the tunnel boundaries are not sharply defined but have peaks in the electron concentration near impurity centers. Effective spin-spin coupling is disrupted if the difference in Knight shifts between neighboring nuclei is more than the "local linewidth." This suggests a spin-diffusion-barrier concept similar to that used to model the paramagnetic spin-lattice interaction. The range of this barrier depends directly on the polarization of the conduction electrons which, in turn, has a Curie-law dependence. In fact, the size of the barrier, $b$, is directly proportional to $(H / T)^{1 / 3}$ and, therefore, dependent on the resonance frequency. ${ }^{13}$ For rapid spin diffusion, $1 / T_{1}$ is proportional to $b^{-3}$ [see Eq. (8)]. Thus, the frequency dependence is $T_{1} \propto \omega_{0}$. This is consistent with the experimental data.

A second model follows a suggestion, by Toyozawa, ${ }^{23}$ that localized magnetic moments are maintained at low temperatures when an impurity conduction band is formed. The spin-lattice relaxation mechanism would, therefore, be similar in nature to that of paramagnetic impurity sites and a frequency dependence would not be unexpected.

Knight-shift measurements are presented in Fig. 5. As noted before, no Knight shifts were detected in the lightly doped samples $A-F$. Clear- ly, the data on samples $J-L$ are in reasonable agreement with Eq. (11) with $K \propto n^{1 / 3}$. This is consistent with the interpretation that these samples are metallic. Below the $N_{\mathrm{cb}}$ concentration, the Knight shift drops off markedly.

Small Knight shifts were detected in samples $G$ and $H$ at 300 and $77^{\circ} \mathrm{K}$; however, no Knight shift was detected in sample $H$ at $4.2^{\circ} \mathrm{K}$. On the other hand a Knight shift was measured in the quasimetallic sample $I$ at $4.2^{\circ} \mathrm{K}$. The onset of a Knight shift at low temperatures seems to be a sensitive indicator of the Mott transition.

The $T_{1}$ data and Knight-shift measurements were used to calculate Korringa products. Figure 6 is a $\log -\log$ plot of $T_{1} T K^{2}$ versus $n$. Here again the three most heavily doped samples satisfy the metallic criteria, with $T_{1} T K^{2}$ being reasonably constant as predicted by Eq. (12). The value $T_{1} T K^{2} \simeq 3.3 \times 10^{-6} \mathrm{sec}^{\circ} \mathrm{K}$ implies an effective Landé $g$ factor of 1.6 as calculated from Eq. (12) using $g=\gamma_{e} h / \mu_{B}$, where $\mu_{B}$ is the Bohr magneton. ${ }^{24}$ This is somewhat lower than the $g=1.7-1.8$ normally measured in CdS. ${ }^{8}$ It should be noted that these reported values of $g$ were measured in rather pure CdS while our data were secured in heavily doped samples.

In the analyses of NMR data discussed thus far, the measurements of conduction-electron concentrations have been indispensable. In fact, some of the conclusions drawn from these analyses are apparent from the electrical measurement data alone. For example, both the Mott transition and the transition associated with the merging of conduction bands can usually be roughly identified from temperature-dependent measurements of the Hall coefficient. Figure 7 is a $\log -\log$ plot of $\left|R_{H}\right|$ versus temperature and contains data on all

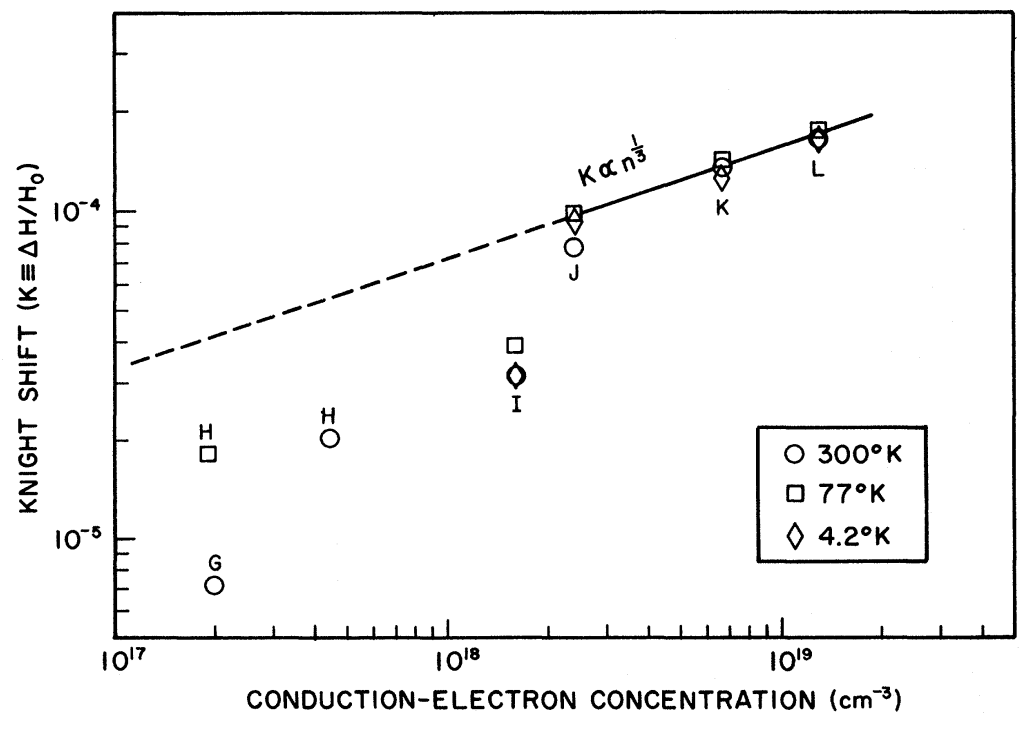

FIG. 5. Knight shift versus conductionelectron concentration. 


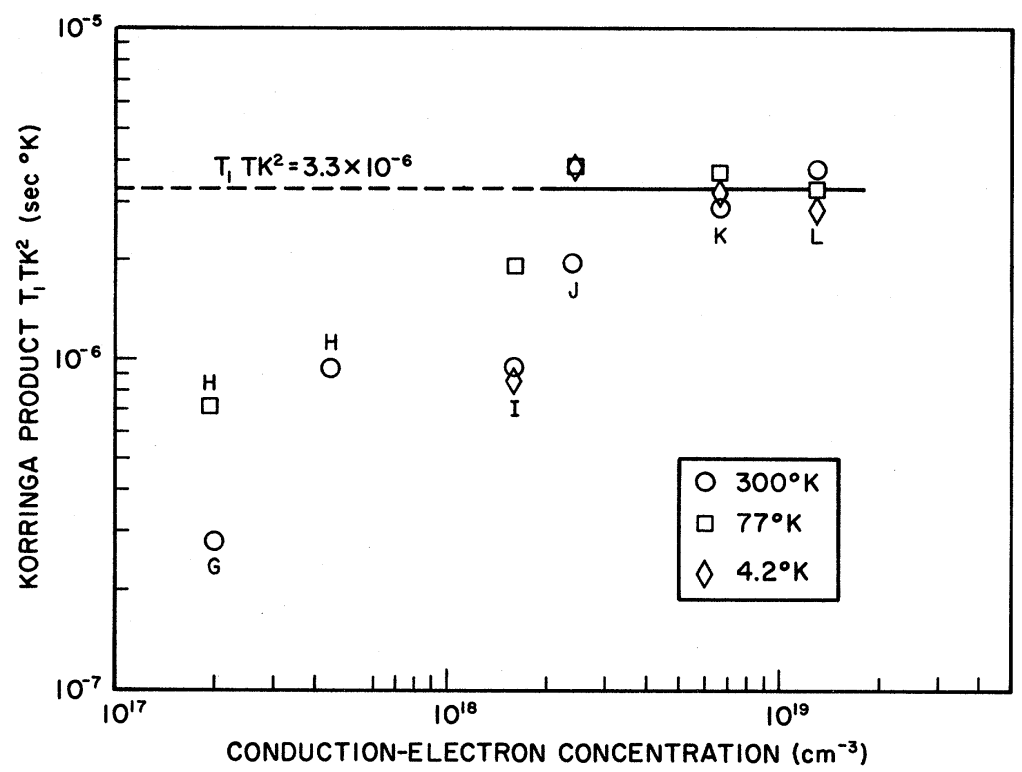

FIG. 6. Korringa product versus conduction-electron concentration.

of the samples $A-L$. From these curves, it is immediately obvious that $\left|R_{H}\right|$ is a monotonically decreasing function of temperature for samples $A-H$. This behavior is typical of semiconductors. The temperature dependence of $\left|R_{H}\right|$ is radically changed in a region bounded by samples $H$ and $I$ such that the latter has only a weak temperature dependence. This is characteristic of a sample which possesses an impurity conduction band. Conwell ${ }^{25}$ has shown that the electron concentration in such a sample is very likely independent of temperature, and the weak temperature dependence of $\left|R_{H}\right|$ is a result of having two conduction bands. For samples $J-L,\left|R_{H}\right|$ is independent of temperature. This indicates that in a region bounded by samples $I$ and $J$, the two conduction bands are merged. Samples $J-L$ have electrical properties which are characteristic of a metal.

One further comment should be made concerning the temperature dependence of $\left|R_{H}\right|$ in sample $I$ : The data secured between 4.2 and $77^{\circ} \mathrm{K}$ were obtained using the cold-finger apparatus previously described. These were not precision measurements and were made only to bracket the range over which $\left|R_{H}\right|$ varies with temperature. The detailed structure in this curve may not be significant. The data do show that a weak temperature dependence is present and that a maximum $\left|R_{H}\right|$ occurs near the liquid-nitrogen temperature of $77^{\circ} \mathrm{K}$. No attempt was made to analyze measurements beyond this point.

\section{SUMMARY}

An NMR study of the semiconductor-to-metal transition in CdS: $\mathrm{Cl}$ has been accomplished. The results are in reasonable agreement with theory and may be summarized as follows:

(i) An impurity conduction band is formed (or the donor electrons become "free") in CdS:Cl when

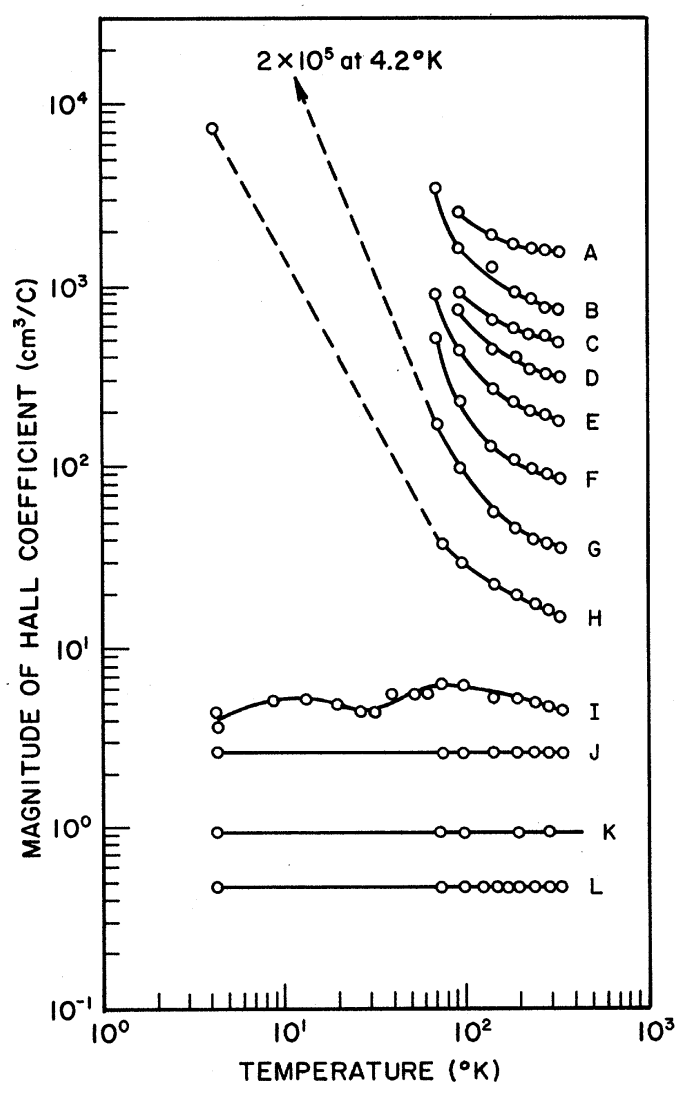

FIG. 7. Magnitude of Hall coefficient versus temperature. 
the conduction-electron concentration $n$ attains a value in the region $5.0 \times 10^{17}<n<1.6 \times 10^{18} \mathrm{~cm}^{-3}$. The predicted value of the impurity concentration is $N_{c}=9 \times 10^{17} \mathrm{~cm}^{-3}$.

(ii) The impurity conduction band and the CdS conduction band are merged (or the electron Fermi level crosses into the CdS conduction band) to complete a semiconductor -to-metal transition in a concentration region $1.6 \times 10^{18}<n<2.4 \times 10^{18} \mathrm{~cm}^{-3}$. The predicted value of $N_{\mathrm{cb}}$ is $N_{\mathrm{cb}} \simeq 4.5 \times 10^{18} \mathrm{~cm}^{-3}$. (Since compensation was not considered these values are in reasonable agreement.)

(iii) For electron concentrations $n<5.0 \times 10^{17}$ $\mathrm{cm}^{-3}$, the electrical properties of CdS: $\mathrm{Cl}$ are basically those of a semiconductor. The spin-lattice relaxation mechanism is due to nondegenerate conduction electrons at $300^{\circ} \mathrm{K}$. At lower temperatures, the conduction electrons are trapped, and the relaxation process is dominated by paramagnetic impurity sites. Knight shifts are very small or negligible.

(iv) For electron concentrations $n>2.4 \times 10^{18}$ $\mathrm{cm}^{-3}$, the electrical properties of CdS: $\mathrm{Cl}$ are basically metallic in nature. The spin-lattice relaxation mechanism is due mainly to degenerate conduction electrons. Knight shifts are observed and consistent with existing theory. The Korringa product is $3.3 \times 10^{-6} \mathrm{sec}^{\circ} \mathrm{K}$. This corresponds to an effective Lande $g$ factor of 1.6. This value is somewhat lower than $g \simeq 1.7-1.8$ which has been reported by most investigators. The discrepancy may be due to the very high doping level used here in contrast to the rather pure CdS used by others.

(v) For electron concentrations in the semiconductor-to-metal transition region, $n \simeq 1.6 \times 10^{18}$ $\mathrm{cm}^{-3}$, the electrical properties of CdS: $\mathrm{Cl}$ are "metal like"; however, they exhibit more than usual temperature dependence. In this region, $\mathrm{CdS}: \mathrm{Cl}$ probably possesses a narrow impurity conduction band located in the band gap just below the CdS conduction band. At $300^{\circ} \mathrm{K}$, the dominant spin-lattice relaxation mechanism is nondegenerate conduction electrons. At lower temperatures, the exact nature of the relaxation mechanism becomes less certain. The observed data are consistent with a model which assumes that nuclear spins in or near impurity band "tunnels" are strongly relaxed by impurity-band conduction electrons and that the remaining spins are then relaxed by rapid spin diffusion. The data may also be interpreted in terms of the existence of localized magnetic moments associated with the impurity conduction band. Either one or both of these mechanisms may be responsible for spin relaxation at low temperatures. Small Knight shifts are observed but existing theories are not adequate to interpret these data.

\section{ACKNOWLEDGMENTS}

We wish to thank D. W. Naas for the preparation of the CdS samples and D. E. Johnson for technical assistance.
*This paper is based upon work submitted by F. D. Adams to the Ohio State University in partial fulfillment of requirements for a $\mathrm{Ph} . \mathrm{D}$. degree.

†Work sponsored by the Aerospace Research Laboratories, Wright-Patterson AFB, Ohio, under Project 7885 and Contract No. F33615-67-C-1027.

${ }^{1}$ R. H. Bube, Photoconductivity of Solids (Wiley, New York, 1960), p. 45.

${ }^{2}$ R. K. Sundfors and D. F. Holcomb, Phys. Rev. 136, 810 (1964).

${ }^{3}$ M. N. Alexander and D. F. Holcomb, Rev. Mod. Phys. $\underline{40}, 815$ (1968).

${ }^{4}$ Michael N. Alexander, Phys. Rev. 172, 331 (1968).

${ }^{5}$ D. Jerome, Rev. Mod. Phys. 40 , 830 (1968).

${ }^{6}$ R. P. Benedict and D. C. Look, Phys. Rev. B 2 , 4949 (1970).

${ }^{7}$ S. Toyotomi and K. Morigaki, J. Phys. Soc. Japan 25, 807 (1968).

${ }^{8} \mathrm{M}$. Neuberger, Air Force Materials Laboratory Report No. DS-124/2E, Wright-Patterson Air Force Base, Ohio 45433, 1967 (unpublished).

${ }^{9}$ N. F. Mott, Proc. Phys. Soc. (London) 62,416 (1949); Can. J. Phys. 34, 1356 (1956); Phil. Mag. $\underline{6}$, 287 (1961); Advan. Phys. 16, 49 (1967).

${ }^{10} \mathrm{~T}$. Matsubara and T. Toyozawa, Progr. Theoret. Phys. (Kyoto) 26, 739 (1961).

${ }^{11}$ D. C. Look and G. G. Wepfer, in II-VI Semiconductor
Compounds, edited by D. G. Thomas (Benjamin, New York, 1967), p. 1222.

${ }^{12} \mathrm{~A}$. Abragam, The Principles of Nuclear Magnetism (Oxford U. P., New York, 1961).

${ }^{13}$ W. E. Blumberg, Phys. Rev. $119,79(1960)$.

${ }^{14}$ N. Bloembergen, Physica $25, \overline{386}(1949)$.

${ }^{15} \mathrm{D}$. Tse and I. J. Lowe, Phys. Rev. 166,279 (1968).

${ }^{16}$ W. D. Knight, Phys. Rev. 76, 1259 (1949).

${ }^{17}$ D. A. Zhogolev, Fiz. Tverd. Tela 9 , 59 (1967) [Sov. Phys. Solid State $\underline{9}, 42$ (1967) ].

${ }^{18}$ Charles P. Slichter, Principles of Magnetic Resonance (Harper and Row, New York, 1963).

${ }^{19} \mathrm{~J}$. Korringa, Physica 16, 601 (1950).

${ }^{20}$ E. H. Putley, The Hall Effect and Related Phenomena (Butterworth, London, 1960).

${ }^{21}$ B. A. Kulp, K. A. Gale, and R. G. Schulze, Phys. Rev. 140, 252 (1965).

${ }^{22} \mathrm{D}$. Jerome, C. Ryter, and J. M. Winter, Physics 2, 81 (1965).

${ }^{23}$ Y. Toyozawa, J. Phys. Soc. Japan 17, 986 (1962).

${ }^{24}$ Actually, when the effective $g$ differs from the freeelectron value, $g_{0}=2$, we have $T_{1} T \propto g_{0}{ }^{-2}$ and $K \propto g_{0} g$ so that $T_{1} T K^{2} \propto g^{2}$. [See, for example, W. E. Blumberg and J. Eisinger, Phys. Rev. 120, 1965 (1960).] Note, however, that it is not correct to set $\gamma_{e}=g \mu_{B} / \hbar$ in Eqs. (2), (3), or (11) unless $g=g_{0}$.

${ }^{25}$ E. M. Conwell, Phys. Rev. $\underline{103}, 51$ (1956). 